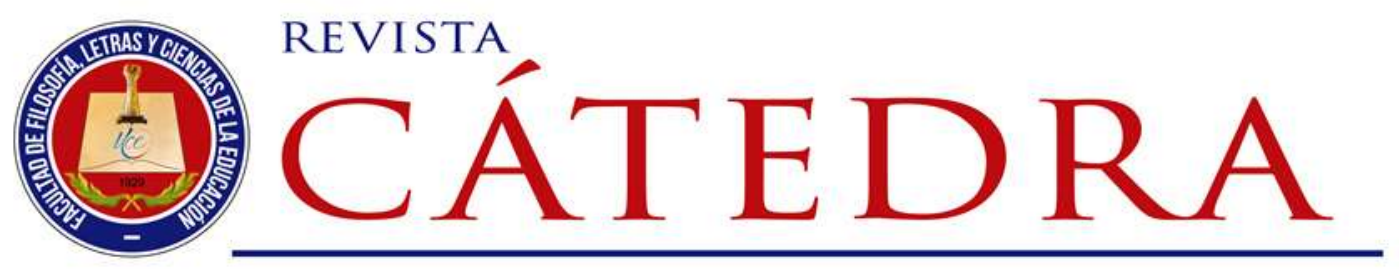

\title{
La Taptana o contador indígena como estrategia de aprendizaje en operaciones matemáticas básicas
}

\section{The Taptana or indigenous counter as a learning strategy in basic mathematical operations}

\author{
Martha Alquinga-Chango \\ Universidad Central del Ecuador, Quito, Ecuador \\ mralquinga@uce.edu.ec \\ https://orcid.org/0000-0001-9746-4079
}

(Recibido: 28/07/2020; Aceptado:30/07/2020; Versión final recibida: 28/08/2020)

Cita del artículo: Alquinga-Chango, M. (2020). La Taptana o contador indígena como estrategia de aprendizaje en operaciones matemáticas básicas. Revista Cátedra, 3(3), 65-87.

\section{Resumen}

El aprendizaje de la Matemática se manifiesta como un problema que necesita ser observado, estudiado y abordado desde múltiples perspectivas para lograr entender el fenómeno y actuar sobre él. Desde diversas teorías educativas se plantea la utilización de materiales didácticos para promover un nivel óptimo en la comprensión de los procesos matemáticos y la adquisición de los conceptos fundamentales de esta ciencia.

La Taptana o contador indígena hace referencia al acervo cultural prehispánico de los antiguos habitantes del actual territorio del Ecuador. Su origen posiblemente se remonta a la cultura Cañari, fase Tacalzhapa, 500 a.C., (Uhle, 1922, p.108). En los años 80 en el Centro de Investigación para la Educación Indígena (CIEI) se recreó este artefacto de cálculo dando como resultado un material didáctico innovador utilizado especialmente, en lo que hasta hace pocos años atrás se conoció como Sistema de Educación Intercultural Bilingüe.

En el presente estudio se indaga sobre la efectividad de la Taptana, como instrumento matemático para la enseñanza-aprendizaje de las operaciones matemáticas básicas. Se trabajó con estudiantes de dos paralelos, A y B, pertenecientes a Cuarto año de Educación General Básica de la Unidad Educativa José María Velasco Ibarra, institución fiscal situada en el centro norte de la ciudad de Quito. La investigación de corte metodológico cualitativo fue desarrollada en los meses de septiembre, octubre y noviembre de 2019.

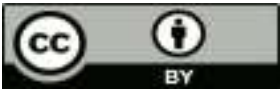


Se logró evidenciar la efectividad de la Taptana. Se concluye que la misma mejora la comprensión y el aprendizaje del sistema decimal y de las operaciones matemáticas básicas.

Palabras clave
Contador indígena, enseñanza aprendizaje, operaciones básicas, Taptana.

\section{Abstract}

The learning of Mathematics manifests itself as a problem that needs to be observed, studied and approached from multiple perspectives in order to understand the phenomenon and act on it. From diverse educational theories, the use of didactic materials is proposed to promote an optimal level in the understanding of mathematical processes and the acquisition of the fundamental concepts of this science.

The Taptana or indigenous accountant refers to the pre-Hispanic cultural heritage of the ancient inhabitants of the current territory of Ecuador. Its origin possibly goes back to the Cañari culture, Tacalzhapa phase, 500 B.C., (Uhle, 1922). In the 80's, the Research Center for Indigenous Education (CIEI) recreated this artifact, resulting in innovative didactic material used especially in what until a few years ago was known as the Intercultural Bilingual Education System.

The present study investigates the effectiveness of the Taptana, as a mathematical instrument for the teaching-learning of basic mathematical operations. We worked with students from two parallel, A and B, belonging to the fourth year of Basic General Education of the José María Velasco Ibarra Educational Unit, a fiscal institution located in the northern center of the city of Quito. The qualitative methodological research was developed in the months of September, October and November 2019.

The effectiveness of the Taptana was demonstrated. It is concluded that it improves the understanding and learning of the decimal system and basic mathematical operations.

\section{Keywords}

Indigenous accountant, teaching learning, basic operations, Taptana.

\section{Introducción}

En el Ecuador como en otros lugares en el mundo, en numerosas ocasiones se ha asociado la enseñanza-aprendizaje de la Matemática con ciertas ideas de complejidad, desagrado, torpeza y desconfianza en la propia capacidad. Otros pensamientos y actitudes similares, probablemente hacen de esta tarea aún más compleja de lo que ya es; en consecuencia, no es extraño observar una especie de temor social, miedo y sentimiento de fracaso ante su estudio. Diferentes evaluaciones locales e internacionales que miden el desempeño de los estudiantes en Matemática, muestran resultados desalentadores.

En América Latina el informe Tercer Estudio Regional Comparativo y Explicativo (TERCE) 2013, desarrollado con la participación de 15 países: Argentina, Brasil, Chile, Colombia, Costa Rica, Ecuador, Guatemala, Honduras, México, Nicaragua, Panamá, Paraguay, Perú, República Dominicana y Uruguay; más el Estado de Nuevo León (México) muestra las deficiencias académicas encontradas en los aprendizajes de la población escolar. En el TERCE se evidencia el complejo problema que representa el aprendizaje de la Matemática en el ámbito escolar. 
En el informe emitido por el Instituto de Evaluación Educativa, INEVAL, se observa que la Matemática sigue teniendo los niveles más bajos en el rendimiento estudiantil.

El 70,9\% de estudiantes de Ecuador no alcanzan el nivel 2, categorizado cómo el nivel de desempeño básico en matemáticas frente al 23,4\% de los estudiantes de países miembros de la Organización para la Cooperación y el Desarrollo Económico, OCDE... Entre los estudiantes con desempeño bajo, el $21 \%$ se encuentra en el nivel $1 \mathrm{~A}$, y solo es capaz de realizar tareas rutinarias en situaciones bien definidas... (Instituto Nacional de Evaluación Educativa, 2018, p. 44).

La matemática constituye una ciencia siempre útil y presente a cada instante de la vida de una persona, es la herramienta estructural de las ciencias exactas y favorece el desarrollo del razonamiento lógico. Lamentablemente, la situación de las matemáticas en las aulas escolares es bastante compleja. La siguiente cita de la UNESCO reitera la dramática situación del aprendizaje de la matemática en la actualidad.

En cuanto a América Latina y el Caribe, 1 de cada 3 niños y adolescentes de la región no puede leer de manera correcta, de acuerdo a lo esperado para su edad, y 1 de cada 2 tiene dificultades serias en matemática. Distintos informes de la UNESCO y de otros organismos internacionales señalan que los niveles mínimos de competencia en matemática a nivel mundial son bajos y muy bajos" (Organización de las Naciones Unidas para la Educación, la Ciencia y la Cultura, UNESCO, 2017).

Es así que en el presente trabajo se analizan los resultados educativos de la aplicación de la Taptana para la enseñanza de la matemática. Se buscó indagar en el proceso de la enseñanza-aprendizaje del sistema decimal y de las operaciones básicas: suma y resta sin y con reagrupación, en estudiantes de Cuarto Año de Educación Básica en la Unidad Educativa "José María Velasco Ibarra", durante los meses de septiembre, octubre y noviembre de 2019.

La enseñanza-aprendizaje de la Matemática constituye un tema investigativo primordial. Es de vital importancia buscar mecanismos que aporten al encuentro entre el estudiante y la Matemática. Se hace necesario un abordaje pedagógico efectivo que permita vivir y valorar la matemática como algo hermoso, divertido y útil, mas no como un problema.

El presente artículo consta de tres partes. En la primera, se presenta una breve referencia histórica sobre la Taptana en su contexto histórico cultural. En la segunda parte consta la metodología utilizada para la aplicación de la Taptana y el proceso mismo de enseñanza aprendizaje desarrollado utilizando la Taptana. Se describen, tanto los instrumentos utilizados, como las actividades y ejercicios realizados. También se mencionan las razones que fundamentan los procesos desarrollados. En la tercera parte se presenta los resultados alcanzados. Para el análisis de datos se utilizó el SPSS, del cual se dice, es el programa estadístico comercial más completo y utilizado a nivel mundial (López y Fachelli, 2015). Finalmente se anotan las conclusiones y recomendaciones que se desprenden de esta investigación.

\section{Revisión de literatura}

\subsection{Breve referencia histórica de la Taptana}

En la provincia del Cañar y en algunos sectores de las provincias de Azuay, Chimborazo, Loja, Morona Santiago, El Oro y Guayas, en Ecuador, se desarrolló la cultura Cañari. Restos 
arqueológicos hallados en el antiguo territorio Cañari, fase Tacalzhapa, 500 a.C. dan cuenta de un hallazgo singular, una piedra de aproximadamente 38 × 25 centímetros, que lleva inscritas hileras laterales de orificios (10) y casilleros subdivididos, a la que se le dio el nombre de Taptana.

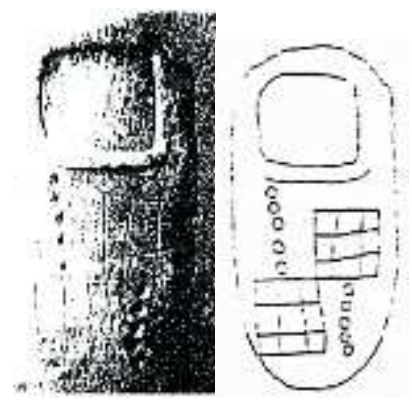

Figura 1. Taptana en piedra y dibujo de la taptana. Fuente: (Butsch, Calero y Muenala, 1998. pág. 6)

Los procesos operativos asociados a la Taptana, probablemente reflejan el pensamiento y forma de vida de los cañaris. Se podría afirmar que en esta piedra cañari se evidencian mecanismos abstractos que se emplearon para resolver problemas de cálculo cotidianos. Las cuadrículas y las circunferencias, según los investigadores del CIEI, podrían hacer referencia al manejo del sistema decimal y también a la cosmovisión andina. Se podría deducir que es posible que en la Taptana tome forma la comprensión ancestral del mundo, en la cual, los datos y el tiempo pueden visualizarse a través del trazado de una espiral, a diferencia de la consabida linealidad que se observa en el razonamiento lógico occidental.

... los granos reemplazan a los elementos reales (animales, productos agrícolas, etc.) que son objeto del cálculo, encontrándose una relación "indirecta" con los referentes que se calculan, o, en otros términos, sustituyendo la realidad objetiva por una realidad representativa... (Yánez, 1996, p. 415).

Así se describe esta particular forma de operar con la Taptana en el mundo indígena andino. Se evidencia como la asignación del significado numérico es arbitraria y a la vez sistemática. Asociar una cantidad a granos de maíz, habas, fréjol fue útil para calcular con rapidez y precisión.

Las investigaciones realizadas por el CIEI motivaron la aplicación de la Taptana para la enseñanza-aprendizaje de la matemática, en el sistema de educación intercultural bilingüe. Además, se desarrollaron algunos proyectos editoriales y se crearon textos escolares en quichua. (Valiente, T. y Küper, W., 1998)

Las raíces culturales de los pueblos andinos presentan grandes aportes científicos en el campo de la agricultura, astronomía y también en la Matemática. La Taptana es un aporte cultural de los Andes al mundo. No se conoce con exactitud el uso de la piedra cañari denominada Taptana. Se sugirió que pudo tratarse de un tablero de juego. Se asocia una posible dirección espiral del movimiento de los granos (al efectuar los cálculos matemáticos) con la cosmovisión andina. Sin embargo, es evidente que aún se necesita un mayor número de investigaciones que den cuenta de la riqueza cultural y científica que dicho artefacto alberga.

La Taptana como recurso didáctico hace décadas ha constado en varios textos escolares del Sistema de Educación Intercultural Bilingüe, (comprendido como el sistema que aglutina a 
los pueblos y nacionalidades del Ecuador). En el libro para la asignatura de Matemática: "Quimsa Yupaicamayuc Camu" publicado en 1989, del autor Humberto Muenala se presentan contenidos matemáticos que van desde el conteo hasta la multiplicación con la utilización de la Taptana.

\subsection{Importancia de la Taptana como material didáctico}

La taptana constituye una muestra de la mente matemática creativa, práctica y compleja de los antiguos habitantes del actual Ecuador, y específicamente del pueblo Cañari en cuyo territorio fueron encontrados vestigios de varias taptanas elaboradas en piedra y madera. Será urgente socializar este hallazgo para nutrir el reconocimiento, respeto y valoración que merece la producción científica de los pueblos ancestrales, en suma, una de las raíces culturales de los ecuatorianos.

La taptana es una especie de ábaco que concreta abstracciones matemáticas y las vuelve manipulables y comprensibles de una manera sencilla y eficaz. Asimilado el mecanismo de su funcionamiento es posible elaborar diversas taptanas en cuanto estructura y materiales de fabricación, de esta forma se podrá contar, en varios contextos, con un importante recurso educativo a la hora de aprender las principales operaciones matemáticas.

\section{Metodología}

La aplicación de la taptana para la enseñanza-aprendizaje de las operaciones matemáticas básicas se realizó en la Unidad Educativa José María Velasco Ibarra, con los estudiantes de los paralelos A y B de Cuarto Año de Educación Básica, de edades comprendidas entre 8 y 9 años. Participaron 60 individuos.

Se debió elaborar tableros de madera de aproximadamente $18 \mathrm{~cm}$. X $25 \mathrm{~cm}$. (taptanas) en cuya superficie fueron grabadas tres hileras verticales de 9 circunferencias. El material se complementó con 19 fichas de colores de aproximadamente $1 \mathrm{~cm}$ de diámetro.

Se trabajó desde un enfoque cualitativo pues se observó el fenómeno educativo desde una mirada humanística. De acuerdo a su finalidad, ésta fue una investigación aplicada. Microsocial, debido al tamaño de la población. Fue una investigación primaria ya que los datos fueron generados por la investigadora. Cuasi experimental porque no se trabajó con grupos equivalentes. Y descriptiva debido a que se relata el proceso de enseñanza aprendizaje de las operaciones matemáticas básicas, en estudiantes de cuarto año de educación básica a través de la taptana.

Para el desarrollo del trabajo de campo se contó con la colaboración de estudiantes de la Facultad de Filosofía, Letras y Ciencias de la Educación, de la Carrera de Pedagogía de la Lengua y la Literatura, las señoritas: Andrea Torres Moreno, y Linda Aguilar Jurado; y el señor Cristian Galarza de la Carrera de Informática.

\subsection{Pruebas aplicadas}

La prueba diagnóstica que se aplicó inicialmente, constó de 12 ítems. Se evaluaron doce destrezas en relación con el manejo de conceptos matemáticos correspondientes a Cuarto Año de Educación General Básica y son las siguientes:

1. Reconoce el valor posicional de un dígito en una cantidad en la posición de las unidades

2. Reconoce el valor posicional de un dígito en una cantidad de 3 cifras en la posición de las decenas 
3. Reconoce el valor posicional de un dígito en una cantidad de 3 cifras en la posición de las centenas

4. Lee y escribe una cantidad de tres cifras

5. Distingue una cantidad mayor de una menor

6. Distingue una cantidad menor de una mayor

7. Opera la suma sin reagrupación de dos sumandos

8. Opera la resta sin reagrupación de dos cantidades

9. Opera la suma con reagrupación a la decena

10. Opera la resta con reagrupación de la decena

11. Resuelve un problema de aplicación de suma con reagrupación

12. Resuelve un problema de aplicación de resta con reagrupación

Inicialmente, se presentó la propuesta a autoridades y docentes de la asignatura de Matemática de la Unidad Educativa J. M. Velasco Ibarra. La aplicación del trabajo con la Taptana se llevó a cabo en dos sesiones semanales, de aproximadamente 80 minutos cada una, durante 10 semanas.

La prueba final se la pasó una vez concluido el proceso anterior. Se buscó evaluar el nivel de adquisición logrado por los estudiantes sobre los mismos conceptos y destrezas matemáticas evaluadas a través de la prueba diagnóstica.

\subsection{La enseñanza de la matemática a través de la Taptana}

El cálculo matemático puede tener distintos sistemas numéricos. Históricamente se conocen los sistemas, vigesimal utilizado por los mayas, quinario, duodecimal, binario, sexagesimal, etc. Se puede decir que es posible organizar el cálculo desde diferentes ideas de agrupación de cantidades. Sin embargo, y dada la amplia expansión del sistema decimal, actualmente, éste es el mecanismo matemático que se emplea en todo el mundo.

El sistema decimal es aquel que usa como referencia principal al número 10 y la posición o ubicación de cada dígito en un orden determinado. Con base en agrupaciones de 10 unidades se crean cada uno de los órdenes con que cuenta el sistema. Así 10 unidades forman una decena, 10 decenas, una centena, 10 centenas forman una unidad de mil y así sucesivamente (Britton y Bello, 1982).

Generalmente el manejo de este contenido matemático, en la escuela regular, se lo realiza desde la memorización de los distintos órdenes nombrados anteriormente y se lo hace a través de lecciones y repeticiones. Frente a esta forma tradicional de aprender matemáticas, se planteó la representación física de las cantidades, su concreción y manipulación para promover una comprensión efectiva del sistema decimal por parte de los estudiantes.

La Taptana es uno de los materiales didácticos más versátiles en cuanto a explicar sensorialmente la formación de cantidades y su significado. Logra mostrar la manera como se conforman las cantidades y cómo se operan. Así surge naturalmente el conocimiento necesario para trasladar los axiomas matemáticos a nuevas situaciones y ejercicios. En la Taptana se hace uso de códigos de color, de formas geométricas u objetos como semillas, (maíz, fréjol y otros granos) y de la ubicación para significar un contenido numérico.

Comprender y adquirir conceptos matemáticos sobre el sistema decimal debería ser un proceso que invite a la reflexión, a la participación activa, al disfrute por conquistar el conocimiento, a la experimentación que despoje al estudiante del miedo al error. Este es un

Licencia Creative Commons Atribución 4.0 Internacional (CC BY 4.0)

Revista Cátedra, 3(3), pp. 65-87, septiembre-diciembre 2020. e-ISSN:2631-2875

https://doi.org/10.29166/catedra.v3i3.2428 
proceso al que se le debería dedicar numerosas horas ya que es la base para el desarrollo de conceptos matemáticos más complejos.

Como se mencionó anteriormente la Taptana inspirada en la antigua piedra cañari, fue diseñada en referencia al sistema decimal. Para la presente investigación se trabajó con la Taptana que contiene tres órdenes: unidad, decena y centena. Es importante indicar que es posible diseñar Taptanas con órdenes hasta el millón y más aún, e incluso otras que expresen números decimales. La lógica implícita en la Taptana permite añadir órdenes o posiciones ascendentes o descendentes de acuerdo a necesidades de trabajo específicas. Ejemplo:

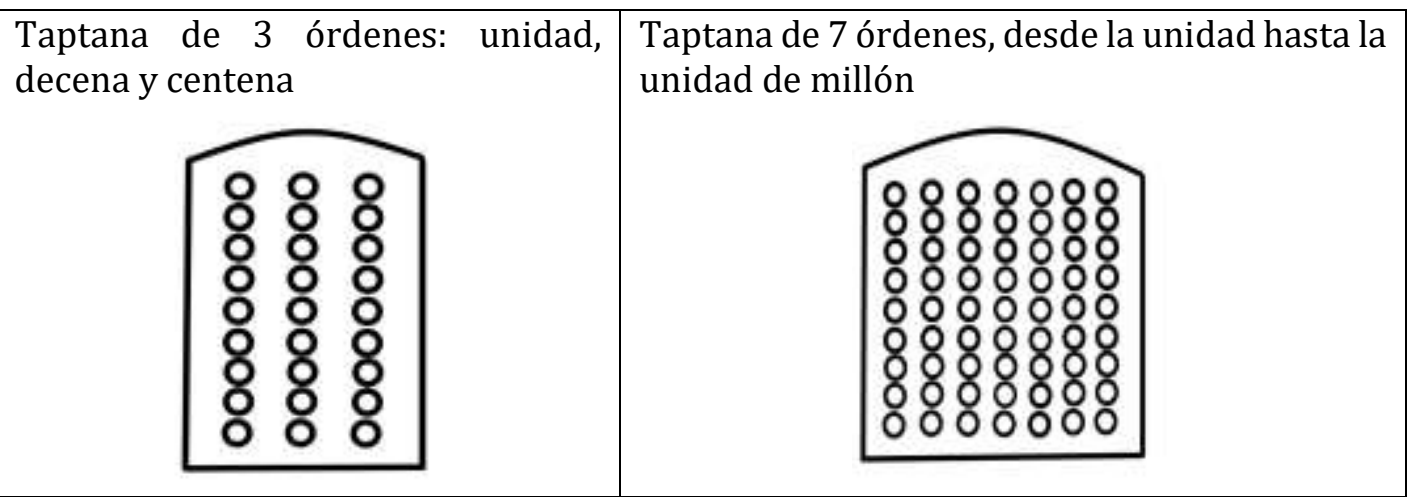

Figura 2: Taptana con tres órdenes: unidades, decenas y centenas. /Taptana con 7 órdenes: unidad, decena centena, unidad de mil, decena de mil, centena de mil y unidad de millón. Fuente: (Elaboración de la autora en base a datos generales tomados de Butsch, Calero y Muenala, 1998)

Es importante mencionar que la Taptana en su estructura presenta la posibilidad de autocorrección ya que existe uno y solo un casillero o lugar para representar cada dígito de una cantidad y al hacerlo de forma errónea se puede observar el fallo con claridad y proceder a corregirlo.

En la presente investigación la enseñanza-aprendizaje de los conceptos matemáticos con la Taptana se planteó a través de las siguientes 4 etapas:

1. Manipulación: colocar las fichas de colores sobre la Taptana para formar cantidades o para realizar operaciones matemáticas.

2. Representación gráfica: dibujar y pintar utilizando lápiz y papel, lo realizado en la etapa anterior con la Taptana.

3. Escritura de la cantidad en números: escribir numéricamente los ejercicios realizados.

4. Escritura de la cantidad en palabras: escribir en palabras los resultados numéricos de los ejercicios realizados.

\subsubsection{Sobre el proceso}

Con la antelación debida se preparó para cada pareja de estudiantes, el siguiente material: una tabla de madera en la cual se encontraba grabada la Taptana, 3 cajas con 19 fichas de colores: verdes, azules y rojas. Pinturas verdes, azules y rojas. Lápices, borradores y sacapuntas. Inicialmente se pensó que cada estudiante realizaría su trabajo de forma individual, sin embargo, y debido a las condiciones poco espaciosas de las aulas y al tipo de muebles, se decidió que se trabajase en parejas.

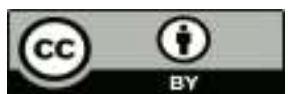


Inicialmente, se dialogó con los estudiantes para reflexionar y asumir las siguientes normas de trabajo:

a) Las mesas de trabajo deben estar vacías para iniciar la tarea de forma organizada y con comodidad

b) El material debe permanecer en orden. (la taptana, las fichas y los recipientes que contienen las fichas),

c) Todos los estudiantes deben tener una libreta, un lápiz y pinturas de los siguientes colores: verde, azul y rojo para realizar los ejercicios.

d) Al finalizar la representación de cada cantidad, las fichas utilizadas se deben regresar a su recipiente para poder realizar el siguiente ejercicio.

e) Las fichas siempre se deben colocar de derecha a izquierda, es decir, se deben representar primero las unidades, luego las decenas y así sucesivamente.

f) Las fichas deben empezar colocándose desde la base de la taptana, nunca desde arriba.

Las cantidades a ser trabajadas en la Taptana fueron seleccionadas tomando en cuenta el grado de dificultad para ir de lo simple a lo complejo, e ir secuencialmente avanzando en los diferentes órdenes del sistema decimal. A través de este proceso se buscó que los estudiantes experimenten el significado del número y procuren ir interiorizando los contenidos matemáticos abstractos.

Para todos y cada uno de los ejercicios, se siguió el siguiente proceso:

Paso 1. Una vez que se explicó el trabajo que se iba a realizar, la investigadora modeló un primer ejercicio, tomando las fichas y colocándolas sobre la Taptana. Al formar las cantidades lo hizo en orden y sin prisa. Después de representar cada cantidad o realizar cada ejercicio se colocó las fichas, una a una, en su respectivo recipiente. Este modelamiento inicial sirvió para que los estudiantes trabajaran con la Taptana de la misma manera. Es importante señalar que los estudiantes estuvieron muy atentos a lo que observaron, quizás un poco más que a la explicación oral.

Paso 2. Se explicó que lo realizado con la taptana, se lo trasladaría a los cuadernos de Matemática. La investigadora dibujó y pintó en el pizarrón a manera de ejemplo el ejercicio en cuestión. En esta etapa se motivó para que el estudiante rememore el proceso realizado, reflexione sobre lo experimentado y lo refiera verbalmente "... Piaget ve las estructuras del conocimiento construidas por el sujeto como resultado de sus propias acciones más que como estructuras procedentes de una fuente externa." (Coll, 1981, p. 27).

Así se evidencia la importancia de la experiencia como mecanismo que permite la asimilación del aprendizaje a las estructuras cognitivas del individuo, como también que este hecho difícilmente ocurriría desde la memorización.

Paso 3. En el pizarrón se escribió en números, el ejercicio hecho con la Taptana.

Paso 4. En el pizarrón, se escribió en palabras la cantidad representada en la Taptana

Terminada la modelación del proceso por parte de la investigadora, se indicó a los estudiantes que desarrollaran con su material, el mismo proceso que acababan de observar.

\subsubsection{La Taptana y la enseñanza-aprendizaje del sistema decimal}

Lista de ejercicios propuestos:

a) $6,2,5,9,4,7,3,8,1$

Licencia Creative Commons Atribución 4.0 Internacional (CC BY 4.0) 


$\begin{array}{llllll}\text { b) } & 12 & 33 & 57 & 61 & 95 \\ \text { c) } & 146 & 271 & 479 & 617 & 834 \\ \text { d) } & 10 & 20 & 50 & 70 & 90 \\ \text { e) } & 250 & 670 & 490 & 710 & 930 \\ \text { f) } & 209 & 302 & 508 & 705 & 908 \\ \text { g) } & 11 & 222 & 444 & 555 & 888 \\ \text { h) } & 262 & 191 & 828 & 454 & 717 \\ \text { i) } & 123 & 246 & 369 & & \end{array}$

Descripción de los ejercicios

a) Cantidades de un solo dígito: se inició la asociación de la posición y el color con la cantidad representada. Este proceso debe realizarse con todos y cada uno de los dígitos para que los estudiantes experimenten la existencia de cada cantidad.

b) Cantidades de dos dígitos: se denotó el lugar que cada dígito ocupa en la Taptana, y en cada cantidad, es decir, tantas unidades y tantas decenas.

c) Cantidades de tres cifras: se utilizaron los tres órdenes de los que constó ésta Taptana. Es importante señalar que se puede iniciar este trabajo con una Taptana que contenga únicamente dos o tres órdenes: unidades, decenas o unidades, decenas y centenas; para luego pasar a una Taptana que incluya los demás órdenes hasta el millón.

d) Cantidades con ceros: se representó decenas puras. La representación del 0 como la ausencia, vacío o falta de una cantidad, llamó la atención de los estudiantes ya que de alguna manera se experimentó la representación de lo que no existe. Este hecho conlleva un mayor grado de dificultad.

e) Cantidades con ceros en la posición de las unidades: se reiteró el significado del cero en una cantidad.

f) Cantidades en cuya formación interviene un cero intermedio: se explicó que el cero puede ubicarse en cualquier posición en una cantidad.

g) Cantidades que resultan divertidas al ser representadas: números que en todos los órdenes tuvieron la misma cantidad de fichas. Esta fue una idea divertida y todo un descubrimiento para los estudiantes, se destacó la idea de lo grato que puede ser representar cantidades.

h) Cantidades simétricas: las figuras que forma una cantidad refuerzan la comprensión del número. Esta idea se puede retomar cuando se trabaje con los demás órdenes del sistema decimal. (unidad de mil, decena de mil... millón).

i) Cantidad en las que se observa una jerarquía interior: el ejercicio fue propicio para comparar cantidades y la forma como están conformadas.

A manera de ejemplo, se muestra la representación gráfica de dos ejercicios:

\begin{tabular}{|l|l|}
\hline Representación gráfica del número 3 & Representación gráfica del número 52 \\
& \\
\hline
\end{tabular}




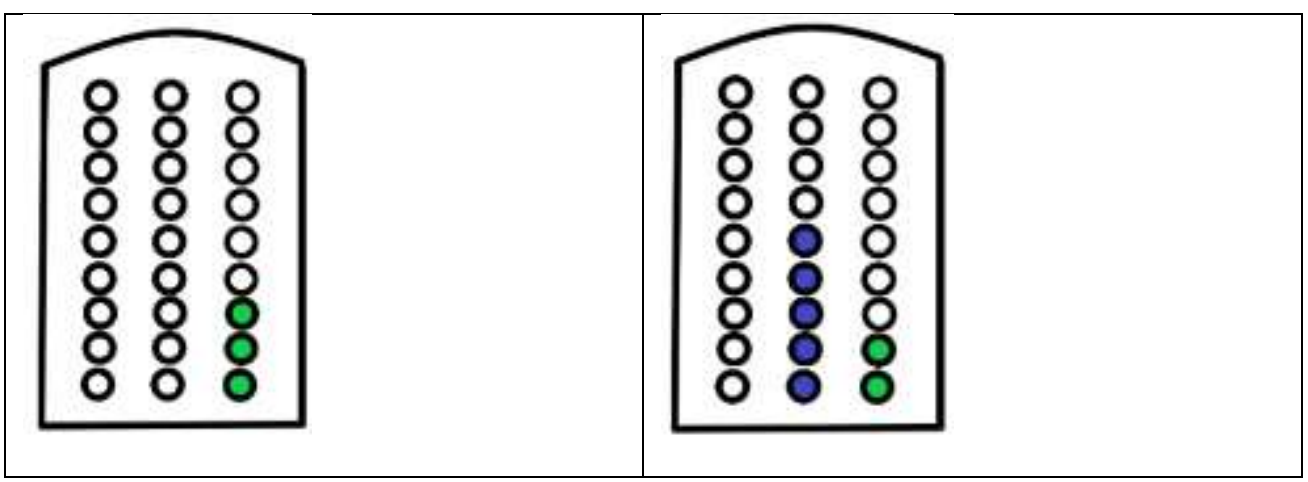

Figura 3: representación gráfica de cantidades en la Taptana

En cada período de clase de aproximadamente 60 minutos, los estudiantes siguieron las instrucciones en la formación de cantidades y lograron hacerlo en orden y con éxito. En ese momento se invitó a sugerir cantidades para que sean representadas por todos los estudiantes de la clase. Esta actividad se convirtió en un reto y una motivación. Fue importante cuidar de no cansar a los estudiantes e ir midiendo los tiempos de atención para no forzar el trabajo y que éste no se torne molesto o aburrido. Se tuvo el cuidado necesario para que cada ejercicio sea realizado sin prescindir de ninguna fase del proceso.

Es importante indicar que se respetó el ritmo de trabajo individual y la estética particular. Cada quien decidió el tamaño y ubicación de las representaciones gráficas de los ejercicios realizados y de otros detalles, al momento de trasladar los ejercicios al cuaderno de Matemática.

\subsubsection{La Taptana y el proceso de enseñanza-aprendizaje de la suma sin reagrupación}

La suma es la operación matemática en la que se juntan dos o más cantidades. Inicialmente y hasta que los estudiantes cobraran confianza y satisfacción al operar la suma fue primordial sumar cantidades sin reagrupar o "llevar". La reagrupación es una idea compleja que necesita una previa experimentación y ejercitación.

Para operar la suma se siguió un proceso que fue de lo simple a lo complejo. Se cuidó el grado de dificultad que debió ser superado cada vez y antes de pasar al siguiente nivel de complejidad.

En cada ejercicio se desarrolló un aprendizaje particular que fue construyendo la comprensión global del significado de la suma.

Lista de ejercicios propuestos:
a) $3+4$,
$5+2$,
$7+1$
b) $11+32$
$16+31$
$54+35$
c) $223+126$
$345+234$
$572+416$
d) $351+10$
$817+120$
$734+200$
e) $406+0$
$718+0$
$583+0$
f) $703+80$
$901+60$
$407+50$
g) $1+3+5$
$4+2+1$
$6+1+2$
h) $31+2$
$83+5$
$47+2$
i) $234+11+1$
$451+12+4$
$352+23+1$
j) $10+20+30$
$40+20+10 \quad 20+30+40$
k) $200+300+400$;
$100+600+200$;
$300+100+400$ 
Descripción de los ejercicios:

a) Sumas de dos cantidades, cada una de ellas de un solo dígito.

b) Sumas de dos cantidades, cada una de ellas compuesta de dos dígitos. Poco a poco los estudiantes fueron experimentando la ocupación de los distintos órdenes en la Taptana. Se fue reforzando la conceptualización del significado de cantidad.

c) Sumas de cantidades compuestas por tres dígitos.

d) Sumas de cantidades que incluyen el cero

e) Sumas de un sumando más cero

f) Sumas con sumandos que incluyen ceros en los distintos órdenes

g) Sumas con tres sumandos de un solo dígito

h) Sumas con un sumando compuesto de dos dígitos y un sumando de un dígito

i) Sumas tres sumandos de tres cifras, dos cifras y una cifra

j) Sumas con tres sumandos de decenas exactas

k) Sumas de tres sumandos de centenas exactas.

A manera de ejemplo, se muestra la representación gráfica de dos ejercicios:

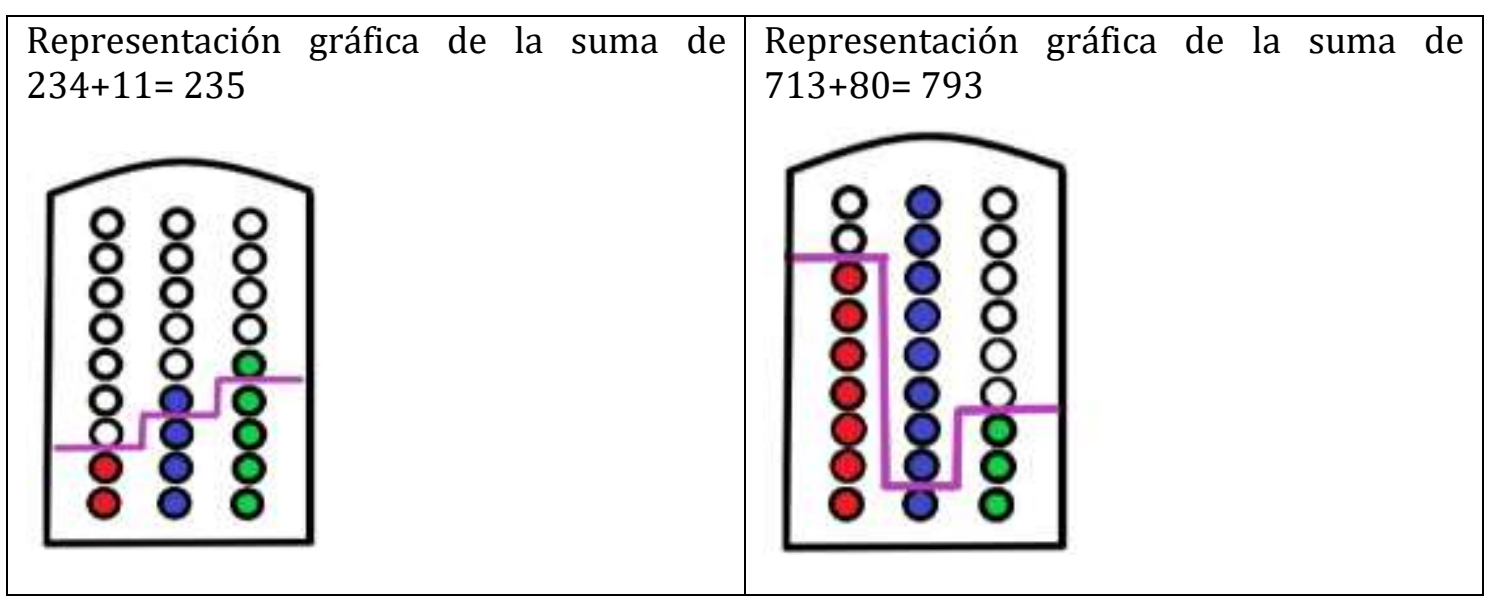

Figura 4: representación de la suma sin reagrupación en la Taptana

\subsubsection{La Taptana y el proceso de enseñanza-aprendizaje de la resta sin reagrupación}

La resta es la operación matemática que complementa la suma y consiste en quitar o retirar una cantidad de otra. Se trabajó en un listado de ejercicios para representar aspectos particulares de la resta y así configurar el aprendizaje integral de esta operación matemática.

Lista de ejercicios propuestos:
a) $9-3 \quad 7-4$
8-5
b) $56-23,83-41$
97-54
c) $489-341 \quad 852-741$
d) $618-10452-30954-50$
e) $60-20 \quad 90-30$
$70-40$
f) $601-0 \quad 189-0$
937-0
g) $893-100 \quad 561-200, \quad 957-300$

Descripción de los ejercicios:

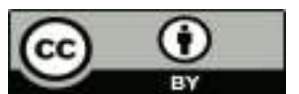


a) Restas donde el minuendo y el sustraendo constaron de un solo dígito

b) Restas donde el minuendo y el sustraendo constaron de dos dígitos

c) Restas donde el minuendo y el sustraendo constaron de tres dígitos

d) Restas donde el minuendo constó de tres dígitos y el sustraendo está formado por decenas exactas

e) Restas donde el minuendo y el sustraendo estuvieron conformados por decenas exactas

f) Restas en las que el sustraendo fue 0

g) Restas donde al minuendo de tres cifras se le debe restar una centena exacta

A manera de ejemplo, se muestra la representación gráfica de dos ejercicios:

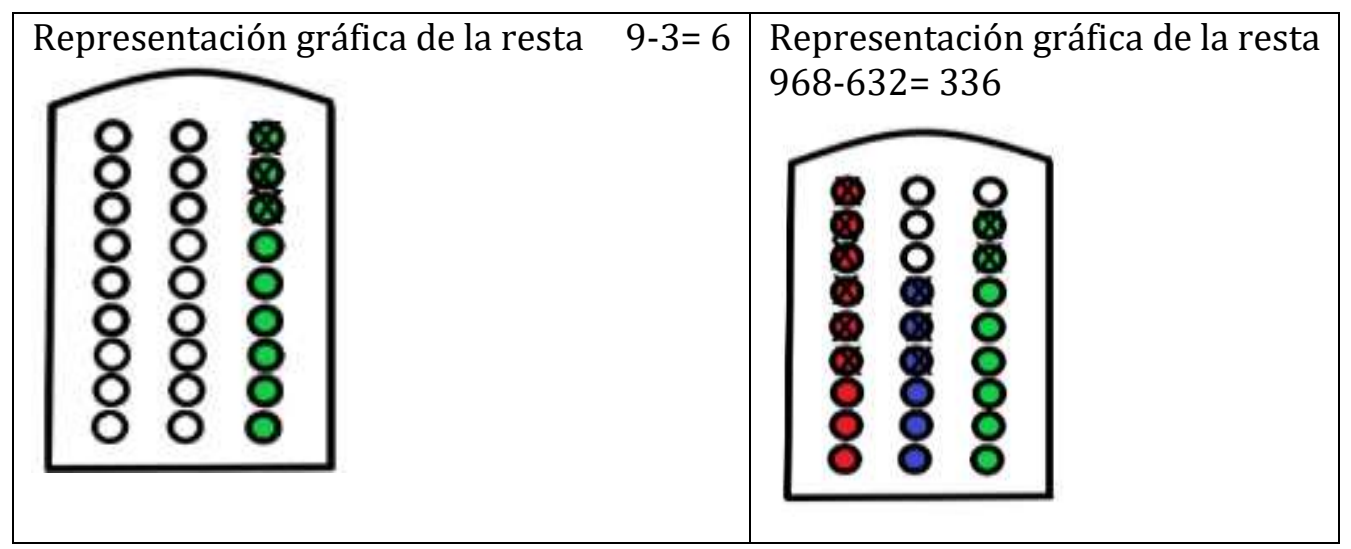

Figura 5: proceso de representación de la resta sin reagrupación en la Taptana

\subsubsection{La Taptana y la enseñanza aprendizaje de la suma con reagrupación}

Una vez superado el aprendizaje de la suma y la resta sin reagrupación fue posible avanzar hacia la suma con reagrupación o "suma llevando". Ésta es la fase del proceso de la adición que suele presentar mayor dificultad debido al nivel de abstracción demandado. Fue importante dedicar el tiempo necesario para brindar las explicaciones y ejemplificaciones requeridas. También se verificó en la práctica, si las instrucciones fueron comprendidas y si se las siguió.

Regularmente, este proceso suele consistir en la repetición verbal del algoritmo aprendido de memoria. Ejemplo:

“... como $5+6=11$, escribo el 1 y llevo $1 \ldots "$... $\begin{gathered}5 \\ \frac{+6}{11}\end{gathered}$

A través de una actividad exclusivamente verbal es difícil saber si el estudiante comprende el significado del algoritmo. Se debería indicar que, al operar esta clase de sumas, lo que se hace es reagrupar los elementos para formar una unidad del siguiente orden superior. En el caso de la suma $5+6$, se ha formado un grupo de más de 10 unidades. Lo que se debe hacer es reagrupar 10 unidades y llevarlas al siguiente orden superior como una decena. Luego se anota lo que resultó, en este caso, una unidad en el orden de las decenas y una unidad en el orden de las unidades. Se obtiene como resultado de esta suma, 11. 
Con la utilización de la Taptana, se visualiza un desequilibrio cognitivo. En la Taptana, en las columnas de unidades, decenas, centenas... solo existen 9 espacios, para 9 fichas y al operar la suma con reagrupación se necesitan 10 o más espacios inexistentes.

El proceso de la suma con reagrupación es propicio para explicar que en el sistema decimal se realizan agrupaciones de 10 elementos en 10 elementos. Así, al tener 10 o más unidades en uno de los órdenes, se debe formar un nuevo grupo de diez elementos que debe trasladarse al siguiente orden superior. En las unidades, cuando se tiene 10 fichas verdes se debe cambiar esas 10 fichas verdes por 1 azul que representa 1 decena. Al tener 10 fichas azules, se debe cambiar por 1 ficha roja que representa 1 centena; y así sucesivamente con los siguientes órdenes de miles, millones, etc.,

El proceso trabajado con la Taptana fue el siguiente:

1. Colocar el primer sumando

2. Colocar el segundo sumando.

3. Ir agrupando y "llevando" al siguiente orden, cada vez que se tengan 10 o más fichas (se pueden tener hasta 19) en el sitio de las unidades o decenas. (en esta investigación se trabajó hasta el orden de las centenas dados los contenidos curriculares para este nivel escolar).

4. Escribir el resultado en números y palabras

Lista de ejercicios propuestos:
a) $7+4$
$8+5$
$9+3$
b) $12+9$
$45+6$
$86+7$
c) $156+5$
$478+3$
$742+9$
d) $26+45$
$64+39$
$73+18$
e) $234+358$
$367+218$
$652+219$
f) $145+371$
$562+281$
$421+293$
g) $256+166$
$278+243$
$589+135$
h) $7+3$
$5+5$
$6+4$
i) $8+12$
$45+55$
$67+33$
j) $380+190$
$120+280$
$670+140$

Descripción de los ejercicios:

a) Sumas entre cantidades de un solo dígito con reagrupación hacia las decenas

b) Sumas entre una cantidad de dos dígitos y una cantidad de un dígito, con reagrupación hacia las decenas

c) Sumas de una cantidad de tres dígitos más una cantidad de un dígito con reagrupación hacia las decenas

d) Sumas entre cantidades de dos dígitos manteniendo la reagrupación de las unidades hacia las decenas

e) Sumas entre cantidades de tres dígitos manteniendo la reagrupación de las unidades hacia las decenas

f) Sumas entre cantidades de tres dígitos con reagrupación de las decenas hacia las centenas y sin reagrupación en las unidades

g) Sumas entre cantidades de tres dígitos con reagrupación en las unidades hacia las decenas y en las decenas hacia las centenas

h) Sumas entre cantidades de un solo dígito para completar una decena exacta 
i) Sumas entre decenas para completar centenas exactas

j) Sumas con cero en las unidades y con reagrupación de las decenas hacia las centenas

A manera de ejemplo, se muestra la representación gráfica de dos ejercicios:

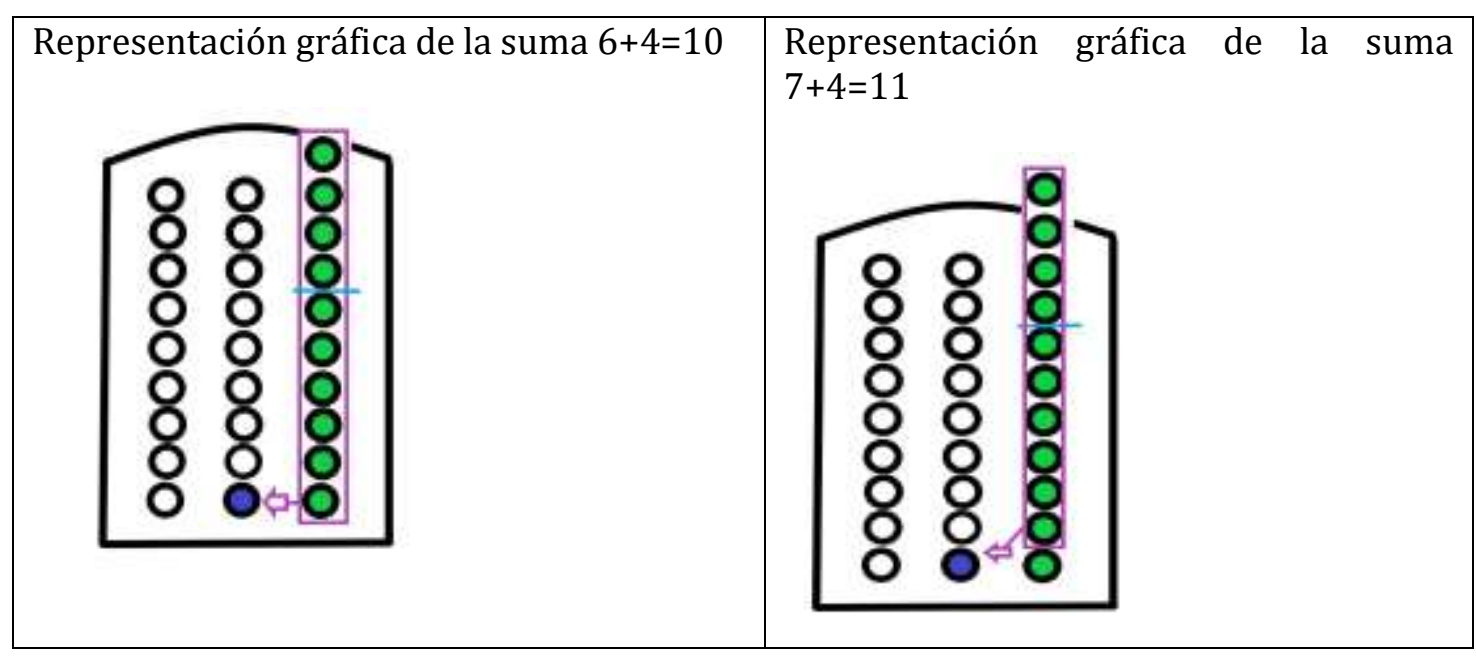

Figura 6: proceso de representación de la suma con reagrupación en la Taptana.

3.2.6 La Taptana y el proceso de la enseñanza-aprendizaje de la resta con reagrupación $\mathrm{Al}$ igual que la suma con reagrupación, la resta con reagrupación resulta un proceso complicado si no se cuida el desarrollo de la comprensión. Al contrastar, la forma tradicional en que suele operarse la resta, con el proceso efectuado con la Taptana, se podrá apreciar la diferencia.

Generalmente, se dice:

\section{2 \\ $\underline{-5}$}

“2 menos 5, no me alcanza, como no me alcanza para quitar 5 de 2, pido prestado 1 y resto".

En la mente de un escolar 8 o 9 años esta idea resulta bastante incompresible debido al nivel de abstracción que requiere entender este planteamiento. Con la Taptana se presenta al estudiante un proceso que explica la expresión "no me alcanza y pido prestado..."

A través de la manipulación de las fichas sobre la Taptana, el estudiante puede darse cuenta del significado de "pedir prestado". Se hace evidente que se procede a desarmar o convertir, una decena tomada del orden inmediato superior, en 10 unidades. El estudiante puede observar las 10 unidades (fichas verdes) contenidas en la decena, ya sueltas y apreciar cada una, como un elemento individual. Esta transformación de la decena en unidades hace posible que se puedan quitar 5 de "2" y así proseguir con la resta.

Lista de ejercicios propuestos:
a) $14-5$
23-6
56-8
b) $63-17$
71-28
83-59
c) $441-256$
$12-54$
863-37
d) $532-143$
751-262
854-165
e) $645-150$
731-240
846- 160

Licencia Creative Commons Atribución 4.0 Internacional (CC BY 4.0) 
Descripción de los ejercicios:

a) Restas donde el minuendo tiene 2 cifras, el sustraendo 1 y se realiza la reagrupación desde la decena.

b) Restas donde el minuendo y el sustraendo constan de 2 cifras. La reagrupación se realiza desde la decena.

c) Restas donde el minuendo tiene 3 cifras, el sustraendo 2 cifras y se realiza la reagrupación desde las decenas.

d) Restas donde el minuendo y el sustraendo tienen tres cifras. Se realiza la reagrupación desde las decenas.

e) Restas donde tanto el minuendo como el sustraendo son cantidades de 3 cifras y se realiza la reagrupación desde la centena.

A manera de ejemplo, se muestra la representación gráfica de dos ejercicios:

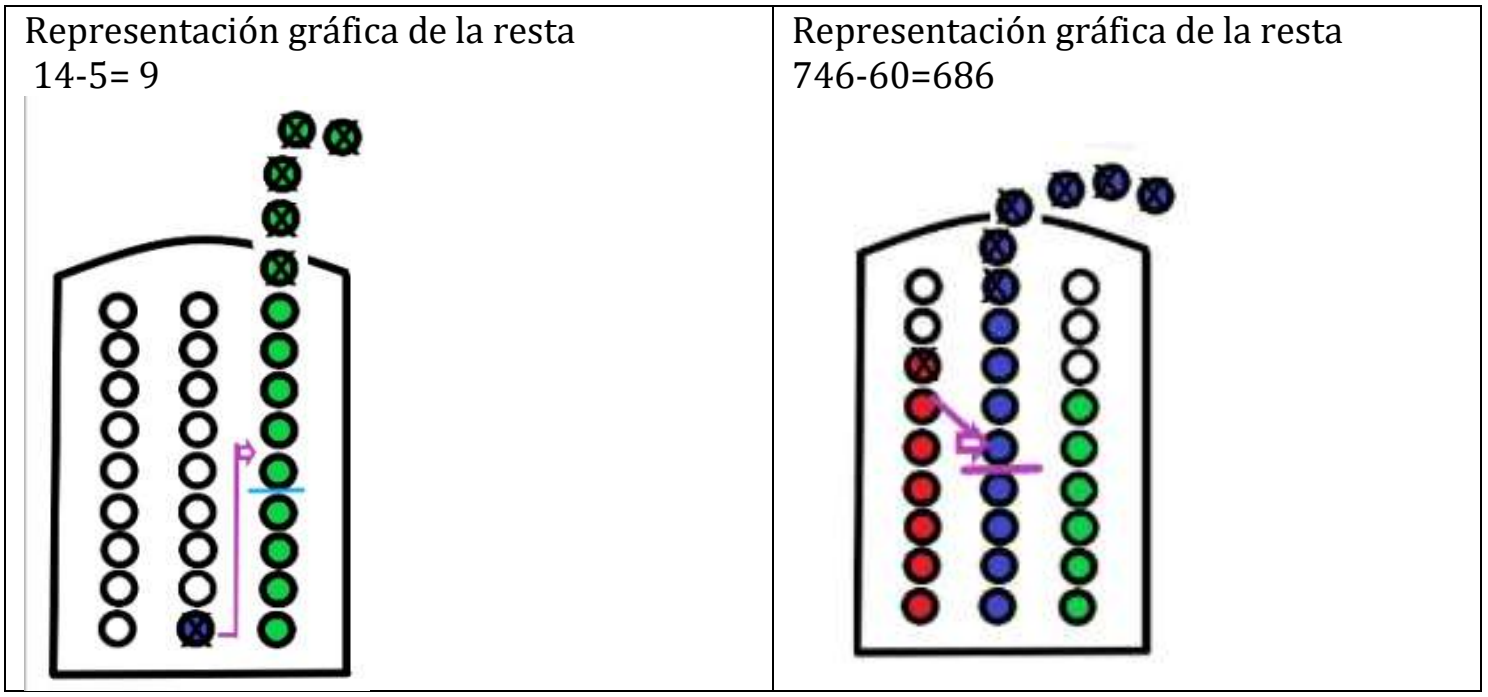

Figura 7: proceso de representación de la resta con reagrupación en la Taptana

\section{Resultados}

Al concluir la etapa de aplicación de la Taptana en el proceso de enseñanza-aprendizaje de las operaciones matemáticas básicas, se aplicó la prueba final para valorar los resultados conseguidos. Para el análisis de datos se utilizó el programa estadístico SPSS. Los estadísticos para la prueba de hipótesis permiten articular predicciones sobre los resultados de la investigación.

Los resultados recogidos se presentan en dos partes. En la primera, se realiza una prueba de hipótesis para muestras independientes. El objetivo de esa aplicación fue conocer si existieron diferencias estadísticas entre los paralelos A y B, en los puntajes totales obtenidos en las pruebas diagnóstica y final.

El segundo análisis corresponde a muestras relacionadas. Se buscó verificar si existió un cambio estadísticamente significativo entre la Evaluación Diagnóstica y la Evaluación Final para cada uno de los ítems y para el puntaje total.

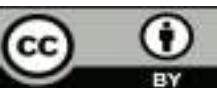


4.1 Prueba de hipótesis para la diferencia de dos poblaciones independientes:

En este estudio tanto el paralelo 4A y 4B son poblaciones independientes y lo que se busca es comprobar si los puntajes entre los dos paralelos son los mismos en el diagnóstico y en la evaluación final:

- Hipótesis nula (Ho):

- El puntaje del paralelo $4 \mathrm{~A}=$ El puntaje del paralelo $4 \mathrm{~B}$

- Hipótesis alternativa (H1):

- El puntaje del paralelo $4 \mathrm{~A} \neq$ El puntaje del paralelo $4 \mathrm{~B}$

- Estadístico de prueba "tobs"

- Criterio de rechazo de Ho:

- Se rechaza Ho si la significancia bilateral<0,05 (en este caso se usa un nivel de significación del 5\%, por eso se anota: 0,05 ).

\begin{tabular}{|c|c|c|c|c|c|}
\hline CURSO & & $\mathrm{N}$ & Media & $\begin{array}{l}\text { Desviación } \\
\text { típ. }\end{array}$ & $\begin{array}{l}\text { Error típ. } \\
\text { De la media }\end{array}$ \\
\hline \multirow{2}{*}{$\begin{array}{l}\text { Puntaje total } \\
\text { prueba } \\
\text { diagnóstica }\end{array}$} & 4to $\mathrm{A}$ & 27 & 7,15 & 2,931 & 0,564 \\
\hline & 4to $B$ & 34 & 6,71 & 2,154 & 0,369 \\
\hline \multirow{2}{*}{$\begin{array}{l}\text { Puntaje total } \\
\text { prueba final }\end{array}$} & 4to $\mathrm{A}$ & 27 & 11,48 & 1,122 & 0,216 \\
\hline & 4to B & 33 & 11,21 & 0,960 & 0,167 \\
\hline
\end{tabular}

Cuadro 1. Prueba de Levene para la igualdad de varianzas

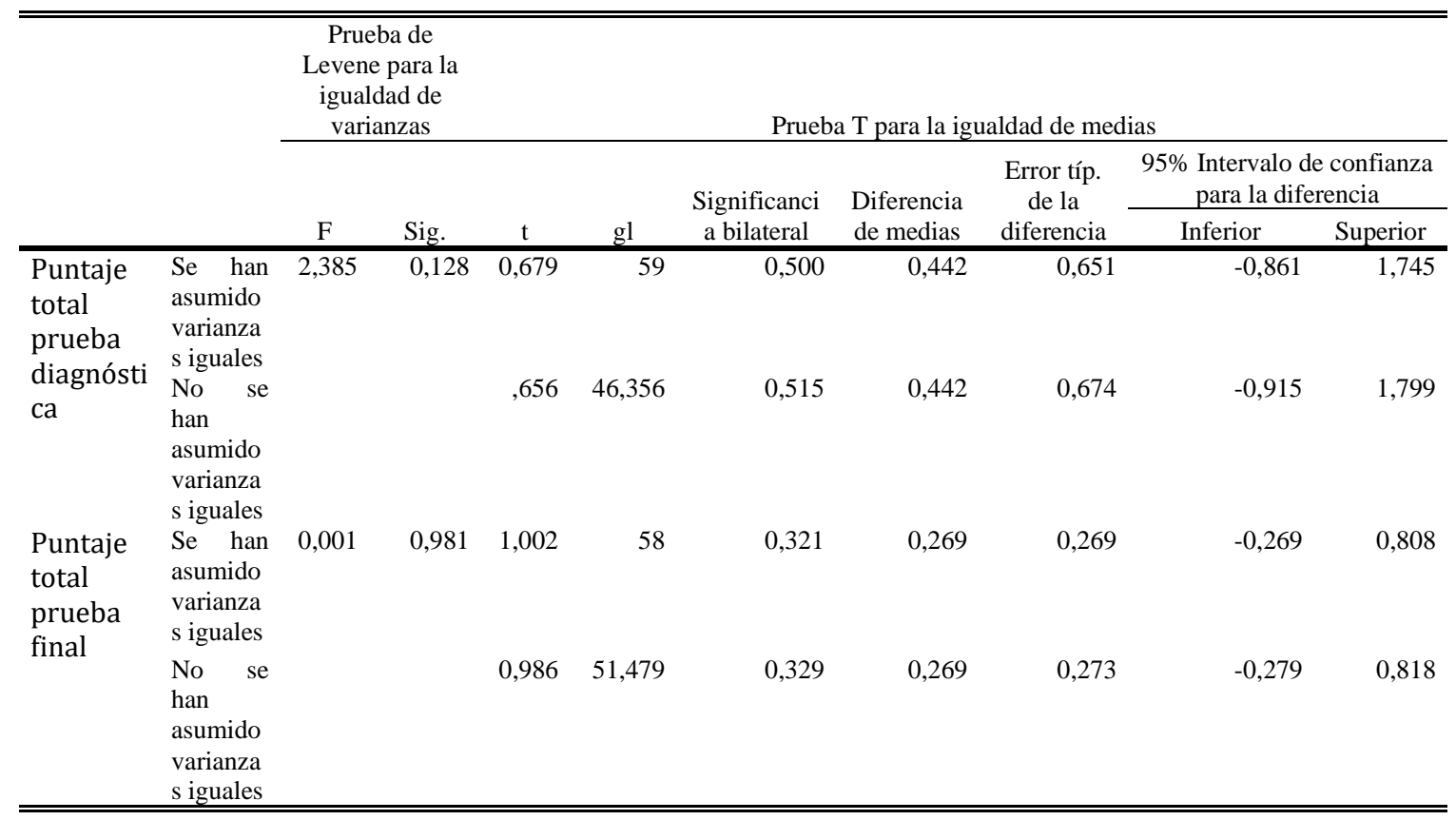

Cuadro 2. Prueba de Levene para la igualdad de varianzas

En ambos casos se asumen varianzas iguales ya que en la prueba de Levene no se rechaza la hipótesis de igualdad de varianzas. Bajo ese supuesto no se rechazan las hipótesis nulas de igualdad de los puntajes en la prueba diagnóstica y la final ya que las significancias bilaterales no son menores al 5\% fijado. 


\subsection{Prueba de hipótesis para muestras relacionadas:}

- Hipótesis nula (Ho):

○ El resultado en el diagnóstico = El resultado final

- Hipótesis alternativa $\left(\mathrm{H}_{1}\right)$ :

- El resultado en el diagnóstico $\neq$ El resultado final

- Estadístico de prueba "tobs"

- Criterio de rechazo de $\mathrm{H}_{0}$ :

- Se rechaza Ho si la significancia bilateral<0,05 (en este caso se usó)

\begin{tabular}{|c|c|c|c|c|c|c|c|c|c|}
\hline & \multicolumn{5}{|c|}{ Diferencias relacionadas } & \multirow[t]{4}{*}{$\mathrm{t}$} & \multirow[t]{4}{*}{$\mathrm{gl}$} & \multirow[t]{4}{*}{$\begin{array}{l}\text { Sig. } \\
\text { (bilat } \\
\text { eral) }\end{array}$} & \multirow[t]{4}{*}{ Decisión } \\
\hline & \multirow[b]{3}{*}{ Media } & \multirow{3}{*}{$\begin{array}{c}\text { Desviació } \\
\text { n típ. }\end{array}$} & \multirow{3}{*}{$\begin{array}{c}\text { Error } \\
\text { típ. de } \\
\text { la } \\
\text { media }\end{array}$} & \multicolumn{2}{|c|}{$\begin{array}{c}95 \% \text { Intervalo de } \\
\text { confianza para la } \\
\text { diferencia }\end{array}$} & & & & \\
\hline & & & & & Superio & & & & \\
\hline & & & & Inferior & $\mathrm{r}$ & & & & \\
\hline $\begin{array}{l}\text { Reconoce el valor posicional de un } \\
\text { dígito en una cantidad en la posición de } \\
\text { las unidades }\end{array}$ & $-0,80$ & 0,40 & 0,05 & $-0,90$ & $-0,70$ & $\begin{array}{c}- \\
15,36\end{array}$ & 59 & 0,000 & Rechazo Ho \\
\hline $\begin{array}{l}\text { Reconoce el valor posicional de un } \\
\text { dígito en una cantidad de } 3 \text { cifras en la } \\
\text { posición de las decenas }\end{array}$ & $-0,75$ & 0,44 & 0,06 & $-0,86$ & $-0,64$ & $\stackrel{-}{13,30}$ & 59 & 0,000 & Rechazo Ho \\
\hline $\begin{array}{l}\text { Reconoce el valor posicional de un } \\
\text { dígito en una cantidad de } 3 \text { cifras en la } \\
\text { posición de las centenas }\end{array}$ & $-0,80$ & 0,40 & 0,05 & $-0,90$ & $-0,70$ & $\stackrel{-}{15,36}$ & 59 & 0,000 & Rechazo Ho \\
\hline Lee y escribe una cantidad de tres cifras & $-0,22$ & 0,42 & 0,05 & $-0,32$ & $-0,11$ & $-4,04$ & 59 & 0,000 & Rechazo Ho \\
\hline $\begin{array}{l}\text { Distingue una cantidad mayor de una } \\
\text { menor }\end{array}$ & $-0,10$ & 0,30 & 0,04 & $-0,18$ & $-0,02$ & $-2,56$ & 59 & 0,013 & Rechazo Ho \\
\hline $\begin{array}{l}\text { Distingue una cantidad menor de una } \\
\text { mayor }\end{array}$ & $-0,12$ & 0,37 & 0,05 & $-0,21$ & $-0,02$ & $-2,43$ & 59 & 0,018 & Rechazo Ho \\
\hline $\begin{array}{l}\text { Opera la suma sin reagrupación de dos } \\
\text { cantidades }\end{array}$ & $-0,05$ & 0,22 & 0,03 & $-0,11$ & 0,01 & $-1,76$ & 59 & 0,083 & $\begin{array}{l}\text { No rechazo } \\
\text { Ho al nivel } \\
\quad 95 \%\end{array}$ \\
\hline $\begin{array}{l}\text { Opera la resta sin reagrupación de dos } \\
\text { cantidades }\end{array}$ & $-0,18$ & 0,47 & 0,06 & $-0,30$ & $-0,06$ & $-3,03$ & 59 & 0,004 & Rechazo Ho \\
\hline $\begin{array}{l}\text { Opera la suma con reagrupación a la } \\
\text { decena }\end{array}$ & $-0,22$ & 0,42 & 0,05 & $-0,32$ & $-0,11$ & $-4,04$ & 59 & 0,000 & Rechazo Ho \\
\hline $\begin{array}{l}\text { Opera la resta con reagrupación de la } \\
\text { decena }\end{array}$ & $-0,52$ & 0,54 & 0,07 & $-0,66$ & $-0,38$ & $-7,46$ & 59 & 0,000 & Rechazo Ho \\
\hline $\begin{array}{l}\text { Resuelve un problema de aplicación de } \\
\text { suma con reagrupación }\end{array}$ & $-0,25$ & 0,60 & 0,08 & $-0,41$ & $-0,09$ & $-3,23$ & 59 & 0,002 & Rechazo Ho \\
\hline $\begin{array}{l}\text { Resuelve un problema de aplicación de } \\
\text { resta con reagrupación }\end{array}$ & $-0,42$ & 0,62 & 0,08 & $-0,58$ & $-0,26$ & $-5,22$ & 59 & 0,000 & Rechazo Ho \\
\hline Puntaje total & $-4,42$ & 2,42 & 0,31 & $-5,04$ & $-3,79$ & $\begin{array}{r}- \\
14,16\end{array}$ & 59 & 0,000 & Rechazo Ho \\
\hline
\end{tabular}

Cuadro 3. Diferencias relacionadas

Del análisis de la tabla de diferencias relacionadas, al contrastar los promedios de la prueba diagnóstica y la prueba final mediante la prueba $\mathrm{T}$ de student, se tiene que: 
Destreza 1: Reconoce el valor posicional de un dígito en una cantidad en la posición de las unidades, se obtuvieron diferencias estadísticamente significativas debido a que el valor de la Sig (bilateral) es menor al p-valor 0.05. Por lo tanto, se descarta la hipótesis nula Ho y se acepta la hipótesis alternativa $\mathrm{H} 1$ la cual indica que hay diferencia significativa en el desarrollo de la destreza matemática: Reconoce el valor posicional de un dígito en una cantidad en la posición de las unidades, después de la aplicación de la Taptana en el proceso de enseñanza-aprendizaje de las operaciones matemáticas básicas: suma y resta.

Destreza 2: Reconoce el valor posicional de un dígito en una cantidad de 3 cifras en la posición de las decenas, se obtuvieron diferencias estadísticamente significativas debido a que el valor de la Sig (bilateral) es menor al p-valor 0.05. Por lo tanto, se descarta la hipótesis nula Ho y se acepta la hipótesis alternativa H1 la cual indica que hay diferencia significativa en el desarrollo de la destreza matemática: Reconoce el valor posicional de un dígito en una cantidad de 3 cifras en la posición de las decenas, después de la aplicación de la Taptana en el proceso de enseñanza-aprendizaje de las operaciones matemáticas básicas: suma y resta

Destreza 3: Reconoce el valor posicional de un dígito en una cantidad de 3 cifras en la posición de las centenas, se obtuvieron diferencias estadísticamente significativas debido a que el valor de la Sig (bilateral) es menor al p-valor 0.05. Por lo tanto, se descarta la hipótesis nula Ho y se acepta la hipótesis alternativa H1 la cual menciona que hay diferencia significativa en el desarrollo de la destreza matemática: Reconoce el valor posicional de un dígito en una cantidad de 3 cifras en la posición de las centenas, después de la aplicación de la Taptana en el proceso de enseñanza-aprendizaje de las operaciones matemáticas básicas: suma y resta

Destreza 4: Lee y escribe una cantidad de tres cifras, se obtuvieron diferencias estadísticamente significativas debido a que el valor de la Sig (bilateral) es menor al p-valor 0.05. Por lo tanto, se descarta la hipótesis nula Ho y se acepta la hipótesis alternativa H1 la cual indica que hay diferencia significativa en el desarrollo de la destreza matemática: Lee y escribe una cantidad de tres cifras, después de la aplicación de la Taptana en el proceso de enseñanza-aprendizaje de las operaciones matemáticas básicas: suma y resta

Destreza 5: Distingue una cantidad mayor de una menor se obtuvieron diferencias estadísticamente significativas debido a que el valor de la Sig (bilateral) es menor al p-valor 0.05. Por lo tanto, se descarta la hipótesis nula Ho y se acepta la hipótesis alternativa H1 la cual menciona que hay diferencia significativa en el desarrollo de la destreza matemática: Distingue una cantidad mayor de una menor, después de la aplicación de la Taptana en el proceso de enseñanza-aprendizaje de las operaciones matemáticas básicas: suma y resta

Destreza 6: Distingue una cantidad menor de una mayor, se obtuvieron diferencias estadísticamente significativas debido a que el valor de la Sig (bilateral) es menor al p-valor 0.05. Por lo tanto, se descarta la hipótesis nula Ho y se acepta la hipótesis alternativa H1 la cual indica que hay diferencia significativa en el desarrollo de la destreza matemática: Distingue una cantidad menor de una mayor, después de la aplicación de la Taptana en el proceso de enseñanza-aprendizaje de las operaciones matemáticas básicas: suma y resta

Destreza 7: Opera la suma sin reagrupación de dos cantidades, no se obtuvieron diferencias estadísticamente significativas debido a que el valor de la Sig (bilateral) es mayor al p-valor 0.05. Por lo tanto, se descarta la hipótesis alternaiva H1 y se acepta la hipótesis nula Ho la cual indica que no existen diferencias significativas en el desarrollo de la destreza matemática: Opera la suma sin reagrupación de dos cantidades, después de la

Licencia Creative Commons Atribución 4.0 Internacional (CC BY 4.0)

Revista Cátedra, 3(3), pp. 65-87, septiembre-diciembre 2020. e-ISSN:2631-2875

https://doi.org/10.29166/catedra.v3i3.2428 
aplicación de la Taptana en el proceso de enseñanza-aprendizaje de las operaciones matemáticas básicas: suma y resta

Destreza 8: Opera la resta sin reagrupación de dos cantidades, se obtuvieron diferencias estadísticamente significativas debido a que el valor de la Sig (bilateral) es menor al p-valor 0.05. Por lo tanto, se descarta la hipótesis nula Ho y se acepta la hipótesis alternativa $\mathrm{H} 1$ la cual indica que hay diferencia significativa en el desarrollo de la destreza matemática: Opera la resta sin reagrupación de dos cantidades, después de la aplicación de la Taptana en el proceso de enseñanza-aprendizaje de las operaciones matemáticas básicas: suma y resta

Destreza 9: Opera la suma con reagrupación a la decena, se obtuvieron diferencias estadísticamente significativas debido a que el valor de la Sig (bilateral) es menor al p-valor 0.05. Por lo tanto, se descarta la hipótesis nula Ho y se acepta la hipótesis alternativa H1 la cual indica que hay diferencia significativa en el desarrollo de la destreza matemática: Opera la suma con reagrupación a la decena, después de la aplicación de la Taptana en el proceso de enseñanza-aprendizaje de las operaciones matemáticas básicas: suma y resta

Destreza 10: Opera la resta con reagrupación de la decena, se obtuvieron diferencias estadísticamente significativas debido a que el valor de la Sig (bilateral) es menor al p-valor 0.05. Por lo tanto, se descarta la hipótesis nula Ho y se acepta la hipótesis alternativa H1 la cual indica que hay diferencia significativa en el desarrollo de la destreza matemática: Opera la resta con reagrupación de la decena, después de la aplicación de la Taptana en el proceso de enseñanza-aprendizaje de las operaciones matemáticas básicas: suma y resta

Destreza 11: Resuelve un problema de aplicación de suma con reagrupación, se obtuvieron diferencias estadísticamente significativas debido a que el valor de la Sig (bilateral) es menor al p-valor 0.05. Por lo tanto, se descarta la hipótesis nula Ho y se acepta la hipótesis alternativa $\mathrm{H} 1$ la cual indica que hay diferencia significativa en el desarrollo de la destreza matemática: Resuelve un problema de aplicación de suma con reagrupación, después de la aplicación de la Taptana en el proceso de enseñanza-aprendizaje de las operaciones matemáticas básicas: suma y resta

Destreza 12: Resuelve un problema de aplicación de resta con reagrupación, se obtuvieron diferencias estadísticamente significativas debido a que el valor de la Sig (bilateral) es menor al p-valor 0.05. Por lo tanto, se descarta la hipótesis nula Ho y se acepta la hipótesis alternativa $\mathrm{H} 1$ la cual indica que hay diferencia significativa en el desarrollo de la destreza matemática: Resuelve un problema de aplicación de resta con reagrupación, después de la aplicación de la Taptana en el proceso de enseñanza-aprendizaje de las operaciones matemáticas básicas: suma y resta

Con la aplicación de las pruebas estadísticas descritas anteriormente, se ha verificado técnicamente que una vez realizado el proceso de enseñanza-aprendizaje a través de la Taptana, se produjo una diferencia que indica una mejora entre las puntuaciones alcanzadas por los estudiantes en la Prueba Inicial o Diagnóstica y la Prueba Final.

Considerando el corto tiempo en el que se desarrolló esta experiencia, (10 semanas) se puede decir que en general este resultado se consideraría positivo. Se evidencia una mejora en las destrezas matemáticas concernientes a la realización de diferentes ejercicios sobre el sistema decimal y la capacidad de operar sumas y restas sin reagrupación y con reagrupación.

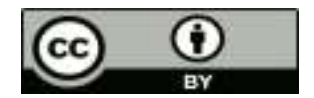




\section{Conclusiones}

Al analizar los resultados de la aplicación de la Taptana para la enseñanza aprendizaje de las operaciones básicas: suma y resta, sin y con reagrupación, se encontró que efectivamente la Taptana es un recurso didáctico propicio para este fin. Se detectó un cambio significativo en cuanto al manejo diestro del sistema decimal, y cambios importantes a nivel de la capacidad de operar sumas.

En cada ejercicio realizado, el estudiante experimentó la concreción del número y la operación matemática; la vivencia de conocer entes abstractos a través de los sentidos del tacto y la vista, posiblemente potenció su comprensión de la matemática. Rousseau 1985 en el Emilio, refirió que los niños razonan muy bien cuando conocen y tienen relación con su presente, también señaló que no se les puede pedir que razonen sobre lo que son incapaces de comprender. La Taptana es una herramienta que dinamiza la comprensión de la lógica implícita en el sistema decimal y en cada una de las operaciones matemáticas; su metodología facilita el tránsito de lo concreto a lo abstracto, el desarrollo de un pensamiento activo y autónomo; y, en consecuencia, un aprendizaje efectivo de las operaciones matemáticas básicas.

Albulquerque, (1953), con respecto a la enseñanza de la Matemática postuló: “... tornar todo o conhecimento concreto" Así, hacer concreto todo conocimiento, ligarlo al uso de los sentidos constituiría una premisa pedagógica para garantizar el aprendizaje. Siendo la matemática una ciencia estructural que fundamenta el desarrollo de numerosos conceptos de la ciencia, es imprescindible que se continúe explorando diversos caminos para mejorar los procesos de enseñanza aprendizaje de la matemática. Se recomienda continuar con la investigación sobre la validez de la Taptana para el aprendizaje de otros conceptos matemáticos como la multiplicación, la división y la raíz cuadrada, dada la riqueza, bajo costo y versatilidad de este material. Además, se considera que es indispensable difundir la utilización de recursos educativos como la Taptana debido a su aporte educativo, cultural y social.

En la Taptana a través de actividades como construir cantidades, operar sumas, restas, reagrupar, se da forma a una serie de conceptos matemáticos. Según Radfor, (2006, citado en D'Amore. 2017. p. 125) la matemática se concreta en la actividad planificada en un tiempo y espacio y sin embargo, sobrepasa la planificación, ya que todas las condiciones implícitas no se pueden determinar por anticipado. Manifiesta que es posible hacerse una idea de lo que ocurrirá en el aula, pero que dicho proceso no es mecánico ni determinístico. Destaca que la dinámica del trabajo en el aula dependerá de cómo los estudiantes y profesores se impliquen en la actividad, de la respuesta entre unos a otros, de las interrelaciones activas en función del saber en general y de las instituciones. Las condiciones para el trabajo propuesto con la Taptana fueron favorables y permitieron alcanzar los resultados esperados.

La enseñanza aprendizaje de las operaciones básicas a través de la utilización de la Taptana es efectiva. Los resultados obtenidos lo demuestran. La falta de interés en la búsqueda de métodos eficaces para brindar el conocimiento matemático a los niños, implica una gran pérdida en su desarrollo integral. La grave situación de los estudiantes de la región en relación con destrezas matemáticas básica, muestra la importancia y urgencia de la realización de sendas investigaciones que apoyen la resolución de esta problemática.

Licencia Creative Commons Atribución 4.0 Internacional (CC BY 4.0)

Revista Cátedra, 3(3), pp. 65-87, septiembre-diciembre 2020. e-ISSN:2631-2875

https://doi.org/10.29166/catedra.v3i3.2428 
Finalmente es importante instar al desarrollo de la investigación educativa ya que es urgente propiciar y promover el encuentro de los mecanismos más idóneos a través de los cuales se despliegue de manera efectiva la enseñanza de la matemática. 


\section{Bibliografía}

Alburquerque, A., (1953). Metodología da Matemática, Río de Janeiro: Ed. Conquista.

Britton, J. y Bello, I., (1982). Matemáticas Contemporáneas, México, D.F. : HARLA S.A. de CV. p.190.

Butsch, G. Calero, V. Muenala, H., (1998). El contador indígena: (Taptana). Quito, Ministerio de Educación y Cultura.

Coll, C., (1981). Psicología genética y educación, Barcelona, oikos-tau, s.a. ediciones.

D’Amore, B., (2017). Enseñanza y aprendizaje de las matemáticas: problemas semióticos, epistemológicos y prácticos, Bogotá, Universidad Distrital Francisco José Caldas.

Educación en Ecuador, Resultados de PISA para el Desarrollo, INEVAL, (2018) http://www.evaluacion.gob.ec/wpcontent/uploads/downloads/2018/12/CIE InformeGeneralPISA18 20181123.pdf

López, P. y Fachelli, S., (2015). Metodología de la Investigación Social Cuantitativa, Barcelona, UAB.

More tan one-half of children and Adolescents Are Not Learning Worldwide, Unesco Institute for Statistics. p.7.

http://uis.unesco.org/sites/default/files/documents/fs46-more-than-half-children-notlearning-en-2017.pdf

Muenala, H., (1989). Quimsa Yupaicamayuc Camu, Quito: Convenio MEC-GTZ-CONAIE.

Rousseau, J., (1985). Emilio o De la educación, Edaf, S.A.

Tercer Estudio Regional Coparativo y Explicativo, TERCE, Unesco, 2013 https://unesdoc.unesco.org/ark:/48223/pf0000227501

Uhle, M., (1922). Sepulturas ricas en oro en la provincia del Azuay, Boletín de la Sociedad Ecuatoriana de Estudios Históricos, 4(12): 108-114, Quito.

UNICEF, 2017. 617 millones de niños y adolescentes no están recibiendo conocimientos mínimos en lectura y matemática.

http://www.unesco.org/new/es/media-

services/single\%20view/news/617 million children and adolescents not getting the mi nimum/

Valiente, T. y Küper, W. (1998). Pueblos Indígenas y Educación, Quito: Abya-Yala.

Yánez, C. (1996). La educación indígena en el Ecuador, Instituto de Capacitación Municipal: Ecuador, Ediciones de la Universidad Politécnica Salesiana. 


\section{Autora}

MARTHA ALQUINGA-CHANGO obtuvo su título de Magíster en Desarrollo de la Inteligencia y Educación por la Pontificia Universidad Católica del Ecuador Sede Ibarra, PUCE-I, es Coordinadora del Área de Etnomatemática en el Instituto de Investigación en Etnociencias, IIEC-UCE.

Actualmente es profesora titular en la Facultad de Filosofía, Letras y Ciencias de la Educación de la Universidad Central del Ecuador. Ha dictado clases en los posgrados: Educación y Desarrollo Social, de la Universidad Tecnológica Equinoccial, UTE y Literatura Infantil y Juvenil de la Universidad Técnica Particular de Loja, UTPL. Su interés investigativo está alrededor de las funciones intelectuales lógicas y la creatividad. Incluye la didáctica de la Matemática a través de elementos ancestrales e innovadores en general, y específicamente de la Taptana, piedra de cálculo o contador indígena, procedente de la cultura Cañari. Ha publicado en la revista Anales de la UCE.

\section{Agradecimientos}

La responsabilidad de cada día mejorar la educación que ofrecemos a niños y jóvenes está en nuestras manos. Dejo constancia de mi agradecimiento a la Dirección de Investigación de la Universidad Central del Ecuador, UCE, y a la Unidad de Investigación Formativa, CIF, por haber hecho posible la realización de la presente investigación. 